doi: $10.13108 / 2020-12-2-3$

\title{
PRODUCTS OF EIGENFUNCTIONS AND WRONSKIANS
}

\author{
A.A. AlLAHVERDYAN, A.B. SHABAT
}

\begin{abstract}
We consider new Wronskian identities found recently in Maikop city. We discuss the relations of these identities with the theory of integrable systems and with the general theory of invertible Darboux transforms for linear differential operators with one independent variable. The object of our study are relations of two Wronskians of orders $N$ and $N^{\prime}>N$ homogeneous with respect to a dilatation group. The elements of the first Wronskian of the order $N$ are arbitrary functions, which enlarges essentially the means of the theory, while the elements of other Wropnskian are formed by the products of these functions of a given order $n \geqslant 2$.

The dilatation group allows us to pass to projective coordinates in the considered quotient of the Wronskians and in particular, to include symmetric functions and polynomials into the considered theory.

The simplest case is naturally $N=2$, in which the second Wronskian turns out to be a power of the original Wronskian and hence, the considered quotient becomes independent of the choice of the elements in the second Wronskian. In this case we obtain new equations for the third and other powers of the eigenfunctions of an one-dimensional Schrödinger equation generalizing known formulae for squares related with the Schwarz derivative and KdV hierarchy.

The case $N=3$ seems to be very interesting from various points of view, but to study it, a further developing of the methods in the projective theory of Wronskians is needed by employing logarithmic derivatives and their higher analogues.
\end{abstract}

Keywords: factorization, Wronski matrix, Schwarz derivative, Riccati equation, Darboux transform.

Mathematics Subject Classification: 35P05; 35B10

\section{WRONSKIANS}

The problem on constructing a differential operator $L$ of order $n \geqslant 2$ by a system of fundamental solutions of the equation $L \varphi_{j}=0, j=\overline{1, n}$ (for the sake of brevity we shall employ a shorthand notation $(j \in[n]))$ is obviously reduced to a linear algebra and the Cramer's rule provides a following formula with the Wronskians for the action of the operator $L(\varphi)$ on an arbitrary smooth function $\varphi$ :

$$
L(\varphi)=\frac{\left\langle\varphi, \varphi_{1}, \ldots, \varphi_{n}\right\rangle}{\left\langle\varphi_{1}, \ldots, \varphi_{n}\right\rangle}, \quad \varphi_{j} \in \operatorname{ker} L
$$

Here we suppose that given functions $\varphi_{1}, \ldots, \varphi_{n}$ form a basis in a $n$-dimensional linear space $\operatorname{ker} L$, and the brackets $\langle\ldots\rangle$ stand for the Wronskian $\left\langle y_{1}, \ldots, y_{m}\right\rangle=\operatorname{det}\left(D_{x}^{k-1}\left(y_{j}\right)\right)$, $j, k=1, \ldots, m$ that is, for the Wronski determinant formed by the derivatives of the considered functions. Formula (1.1) and its specifications, see 3.2 below, replace the factorization of a usual polynomial into linear factors and play a similar role once we specify the structure of the kernel of the considered differential operator $L$.

A.B. Shabat, A.A. Allahverdyan, Products of eigenfunctions and Wronskians.

(C) Shabat A.B., Allahverdyan A.A. 2020.

Submitted February 7, 2020. 
Employing the known properties of the determinants and the Leibnitz formulae, we find that

$$
y_{j}(x)=a(x) \hat{y}_{j}(x), \quad \text { for all } j \Rightarrow\left\langle y_{1}, \ldots, y_{m}\right\rangle=a^{m}\left\langle\hat{y}_{1}, \ldots, \hat{y}_{m}\right\rangle .
$$

In terms of the differential operator (1.1) this operation coincides with the adjoint operation:

$$
L \Leftrightarrow \widetilde{L}, \quad L=\frac{1}{a} \cdot \widetilde{L} \circ a .
$$

We recall that employing adjoining 1.2 in initial formula 1.1 , we can get rid of the denominator by letting $\left\langle\varphi_{1}, \ldots, \varphi_{n}\right\rangle=1$. The highest coefficients in the differential operator $L$ still remains unit but in addition, we vanish the next coefficient at the derivative $\varphi^{(n-1)}$ of order $n-1$. For the Wronskians with $a=1 / \varphi_{n}$, operation 1.2 corresponds to passing to homogeneous coordinates and their logarithmic derivatives in the Wronski matrix:

$$
\begin{gathered}
w_{n}(\vec{\psi}) \stackrel{\text { def }}{=} \frac{\left\langle\psi_{1}, \psi_{2}, \ldots, \psi_{n}\right\rangle}{\psi_{1} \psi_{2} \cdots \psi_{n}}=(-1)^{n-1} \operatorname{det}\left(\begin{array}{cccc}
g_{1} & g_{2} & \ldots & g_{n-1} \\
g_{1}^{\prime}+g_{1}^{2} & g_{2}^{\prime}+g_{2}^{2} & \ldots & g_{n-1}^{\prime}+g_{n-1}^{2} \\
\ldots & \ldots & \ldots & \ldots
\end{array}\right), \\
g_{j}=\left(\log \psi_{j}\right)_{x}-\left(\log \psi_{n}\right)_{x}=\frac{\left\langle\psi_{j}, \psi_{n}\right\rangle}{\psi_{j} \psi_{n}}, \quad j \in[n-1]
\end{gathered}
$$

Lemma 1.1. A transformed Wronskian $w_{n}(\vec{\psi})$ is a homogeneous anti-symmetric polynomial of degree $\frac{1}{2} n(n-1)$ on $n-1$ differential variables $g_{j}, j \in[n-1]$.

Indeed, see [3], as $g=\phi^{\prime} / \phi=(\log \phi)_{x}$, the following differentiation gives:

$$
\frac{\phi^{\prime \prime}}{\phi}=g^{\prime}+g^{2}, \quad \frac{\phi^{\prime \prime \prime}}{\phi}=g^{\prime \prime}+3 g g^{\prime}+g^{3}, \quad \frac{\phi^{(4)}}{\phi}=g^{(3)}+4 g g_{x x}+3 g_{x}^{2}+6 g^{2} g_{x}+g^{4} \ldots
$$

\section{SCHWARZ DERIVATIVE}

Aforementioned formula (1.4) for the Wronskians is a convenient way for the finding differential equations for homogeneous momenta of squares and cubes of the solutions to second order differential equations ${ }^{1} \varphi^{\prime \prime}=u(x) \varphi$. For the squares the answer is known, but to demonstrate a general scheme, we provide detailed calculations in this case as well.

Let $\varphi_{j}^{\prime}=f_{j} \varphi_{j}, f_{j}^{\prime}+f_{j}^{2}=u(x), j \in[2]$ and $\psi_{3}=\varphi_{1} \varphi_{2}$. Then

$$
\psi_{3}^{\prime}=\left[f_{1}+f_{2}\right] \psi_{3}, \quad \psi_{3}^{\prime \prime}=2\left[u+f_{1} f_{2}\right] \psi_{3}, \quad \psi_{3}^{\prime \prime \prime}=2\left[u^{\prime}+2 u\left(f_{1}+f_{2}\right)\right] \psi_{3},
$$

and substituting corresponding formulae for $\psi_{1}=\varphi_{1}^{2}$ and $\psi_{2}=\varphi_{2}^{2}$ into (1.1), we find that

$$
\frac{\left\langle\psi, \psi_{1}, \psi_{2}, \psi_{3}\right\rangle}{\psi_{1} \cdot \psi_{2} \cdot \psi_{3}}=\left(\begin{array}{cccc}
\psi & 1 & 1 & 1 \\
\psi^{\prime} & 2 f_{1} & 2 f_{2} & f_{1}+f_{2} \\
\psi^{\prime \prime} & 2 u+2 f_{1}^{2} & 2 u+2 f_{2}^{2} & 2 u+2 f_{1} f_{2} \\
\psi^{\prime \prime \prime} & 2 u^{\prime}+8 u f_{1} & 2 u^{\prime}+8 u f_{2} & 2 u^{\prime}+4 u\left(f_{1}+f_{2}\right)
\end{array}\right) .
$$

Finally we obtain that in the case of "squares" of solutions to the equation $\varphi^{\text {" }}=u(x) \varphi$, formula (1.1) reads as

$$
L(\psi)=\frac{\left\langle\psi, \psi_{1}, \psi_{2}, \psi_{3}\right\rangle}{\left\langle\psi_{1}, \psi_{2}, \psi_{3}\right\rangle}=\psi^{\prime \prime \prime}-4 u \psi^{\prime}-2 u^{\prime} \psi,
$$

and as $\psi_{1}=\varphi_{1}^{2} \varphi_{2}, \psi_{2}=\varphi_{1} \varphi_{2}^{2}, \psi_{3}=\varphi_{1}^{3}, \psi_{4}=\varphi_{2}^{3}$, we have:

$$
L(\psi)=\frac{\left\langle\psi, \psi_{1}, \psi_{2}, \psi_{3}, \psi_{4}\right\rangle}{\left\langle\psi_{1}, \psi_{2}, \psi_{3}, \psi_{4}\right\rangle}=\psi^{\prime \prime \prime \prime}-10\left(u \psi^{\prime \prime}+u^{\prime} \psi^{\prime}\right)-3\left(u^{\prime \prime}-3 u^{2}\right) \psi
$$

\footnotetext{
${ }^{1}$ as well as for the squares and cubes of solutions to equations with a spectral parameter $u \equiv u-\lambda$
} 
In the latter case, the role of formula (2.1) for the derivatives of the product $\varphi_{1} \varphi_{2}$ is played by the following formulae for the derivatives of the function $\psi_{1}=\varphi_{1}^{2} \varphi_{2}$ :

$$
\begin{gathered}
\frac{\psi_{1}^{\prime}}{\psi_{1}}=2 f_{1}+f_{2}, \quad \frac{\psi_{1}^{\prime \prime}}{\psi_{1}}=3 u+2 f_{1}^{2}+4 f_{1} f_{2}, \quad \frac{\psi_{1}^{\prime \prime \prime}}{\psi_{1}}=3 u^{\prime}+7 u\left(2 f_{1}+f_{2}\right)+6 f_{1}^{2} f_{2}, \\
\frac{\psi_{1}^{\prime \prime \prime \prime}}{\psi_{1}}=3 u^{\prime \prime}+21 u^{2}+10 u^{\prime}\left(2 f_{1}+f_{2}\right)+u\left(20 f_{1}^{2}+40 f_{1} f_{2}\right) .
\end{gathered}
$$

In order to compare with a usual deducing, see, for instance, [6], the equation for the squares of the eigenfunctions of the Schrödinger operator, we multiply equation (2.2) by $\psi$. After integrating, a third order equation $L \psi=0$ is reduced to

$$
C(\lambda)=\psi_{x}^{2}+4(u-\lambda) \psi^{2}-2 \psi_{x x} \psi
$$

where $C(\lambda)$ is an integration constant and $\lambda$ is an additional parameter ${ }^{1}$. As $\psi=\varphi_{1} \varphi_{2}$, formula (2.1) establishes a relation of between the integration constant and the Wronskian $w=\left\langle\varphi_{1}, \varphi_{2}\right\rangle$ :

$$
C(\lambda)=\left(f_{1}-f_{2}\right)^{2}=\left\langle\varphi_{1}, \varphi_{2}\right\rangle^{2} .
$$

On the other hand, $(\log \psi)_{x}=\left(\log \varphi_{1}\right)_{x}+\left(\log \varphi_{2}\right)_{x}=f_{1}+f_{2}$, and hence,

$$
f_{1}=\frac{\psi_{x}-w}{2 \psi}, \quad f_{2}=\frac{\psi_{x}+w}{2 \psi}
$$

Formulae (2.6) hence map a solution of equation $(2.4)$ with Schwarz derivative into a pair of solutions to Ricatti equation, or, in other words, formulae (2.6) and (2.2) establish the equivalence of equation (2.4) to the Ricatti equation $f_{x}+f^{2}=u-\lambda$.

Remark 1. We recall that the problem on constructing solution $\psi$ to equation (2.4) in an explicit form employs an original assumption on a polynomial dependence of a sought function $\psi=\psi(x ; \lambda)$ on the parameter $\lambda$ :

$$
\psi=\lambda^{n}+a_{1}(x) \lambda^{n-1}+\cdots+a_{n}(x) .
$$

In the simplest case $n=1$ the substitution $\psi=\lambda+a_{1}(x)$ in equation (2.4) leads us to the formulae:

$$
C(\lambda)=-4\left(\lambda^{3}+\alpha_{1} \lambda^{2}+\alpha_{2} \lambda+\alpha_{3}\right), \quad u=2 a_{1}-\alpha_{1}
$$

and to a known first order differential equation for the elliptic Weierstrass function:

$$
a_{x}^{2}=C(-a), \quad\left(a \equiv a_{1}(x)\right) .
$$

As $n>1$, the degree of the polynomial $C(\lambda)$ in the left hand side in equation (2.4) is replaced by $2 n+1$, while equation (2.8) transforms into the system of Dubrovin equation [6] for $n$ roots of polynomial (2.7). The coefficients $a_{j}(x)$ of this polynomial (2.7) can be found one by one employing the differential operator $L$ in formula $(2.2)$ :

$$
4 a_{j+1}^{\prime}+L\left(a_{j}\right)=0, \quad a_{0}=1, \quad L=D^{3}-4 u D-2 u_{x} .
$$

Integrated forma (2.4) allows us to express $a_{j}(x)$ in terms of the potential $u(x)$ and its derivatives. For instance, up to lower terms,

$$
8 a_{2}=3 u^{2}-u_{2}, \quad 32 a_{3}=u_{4}-4 u u_{2}+u_{1}^{2}+10 u^{3}, \quad\left(u_{1}=u_{x}, u_{2}=u_{x x}, \ldots\right) .
$$

While substituting homogeneous monomials $\varphi_{1}^{j} \varphi_{2}^{k}=j+k=m$ into general formula (1.1) it is natural to expect that the coefficients of the obtained operator can depend on a particular choice of the basis $\varphi_{1}$ and $\varphi_{2}$. However, this is not so and in our opinion, an explanation for a found invariance in the case of the fourth order $(2.3)$ is provided by the following theorem.

\footnotetext{
${ }^{1}$ the substitution $u \leftrightarrow u-\lambda$ is allowed by formulae $(2.2)$
} 
Theorem 2.1. Let $\varphi_{1}$ and $\varphi_{2}$ be arbitrary smooth functions and

$$
\psi_{1}=\varphi_{1}^{3}, \quad \psi_{2}=\varphi_{1}^{2} \varphi_{2}, \quad \psi_{3}=\varphi_{1} \varphi_{2}^{2}, \quad \psi_{4}=\varphi_{2}^{3} .
$$

Then the identity holds:

$$
\frac{\left\langle\psi_{1}, \psi_{2}, \psi_{3}, \psi_{4}\right\rangle}{\left(\left\langle\varphi_{1}, \varphi_{2}\right\rangle\right)^{6}}=12
$$

Proof. Applying transformation (1.2), cf. formula (1.4), in the numerator we replace $\psi_{j} \rightarrow \psi_{j} / \psi_{4}$ :

$$
\frac{\left\langle\psi_{1}, \psi_{2}, \psi_{3}, \psi_{4}\right\rangle}{\left(\left\langle\varphi_{1}, \varphi_{2}\right\rangle\right)^{6}}=\operatorname{det}\left(\begin{array}{cccc}
1 & 1 & 1 & 1 \\
g_{1} & g_{2} & g_{3} & 0 \\
g_{1}^{\prime}+g_{1}^{2} & g_{2}^{\prime}+g_{2}^{2} & g_{3}^{\prime}+g_{3}^{2} & 0 \\
g_{1}^{\prime \prime}+3 g_{1} g_{1}^{\prime}+g_{1}^{3} & g_{2}^{\prime \prime}+3 g_{2} g_{2}^{\prime}+g_{2}^{3} & g_{3}^{\prime \prime}+3 g_{3} g_{3}^{\prime}+g_{3}^{3} & 0
\end{array}\right),
$$

where $g_{j}$ stand for the logarithmic derivatives of the functions $\psi_{j} / \psi_{4}$. Thus,

$$
g_{1}=3\left(f_{1}-f_{2}\right), \quad g_{2}=2\left(f_{1}-f_{2}\right), \quad g_{3}=f_{1}-f_{2},
$$

where $f_{j}=\left(\log \varphi_{j}\right)^{\prime}, j=1,2$. Substituting these formulae into the above determinant gives identity 2.10) after calculating like terms.

Theorem 2.1 can be also generalized for the case $\operatorname{deg} \varphi_{1}^{j} \varphi_{2}^{k}=j+k=4$, and we obtain

$$
\frac{\left\langle\psi_{1}, \psi_{2}, \psi_{3}, \psi_{4}, \psi_{5}\right\rangle}{\left(\left\langle\varphi_{1}, \varphi_{2}\right\rangle\right)^{10}}=288=2^{5} 3^{2} .
$$

More precisely, we apply formula (1.4) and we find that in this case

$$
\frac{w_{5}(\vec{\psi})}{g_{1} \cdots g_{4}}=\left(\begin{array}{cccc}
1 & 1 & 1 & 1 \\
g_{1} & g_{2} & g_{3} & g_{4} \\
g_{1}^{2} & g_{2}^{2} & g_{3}^{2} & g_{4}^{2} \\
g_{1}^{3} & g_{2}^{3} & g_{3}^{3} & g_{4}^{3}
\end{array}\right)=\prod_{i>j}\left(g_{i}-g_{j}\right)+\ldots
$$

where the dots stand for the terms in formulae 1.5 involving the derivatives $g_{j}$. Similarly to 2.11 we find:

$$
g_{1}=4 g, \quad g_{2}=3 g, \quad g_{3}=2 g, \quad g_{4}=g=\left(\log \frac{\varphi_{1}}{\varphi_{2}}\right)_{x} .
$$

It is easy to see that formula 2.12 follows 2.13 and 2.14 as $g_{j}^{\prime}=0$, that is, for exponential functions $\varphi_{1}$ and $\varphi_{2}$. In a general case, the vanishing of additional terms with the derivatives is to be checked straightforwardly beginning with higher derivatives, cf. [3]), employing explicit formulae (1.5).

In case (2.13), the operator $L$ similar to $(2.2)$ and $(2.3)$ is of the fifth order. Interesting questions on applications of these operators and their relations with the Schwarz derivatives and $\mathrm{KdV}$ like equations remain open.

2.1. Exponential case. The problem on squares of the eigenfunctions of the Schrödinger operator and a related identity

$$
\frac{\left\langle\varphi_{1}^{2}, \varphi_{1} \varphi_{2}, \varphi_{2}^{2}\right\rangle}{\left\langle\varphi_{1}, \varphi_{2}\right\rangle^{3}}=2
$$

are to be modified in the case of the third order operators with a codimension of the zero-space equalling to 3 . Indeed, in the case of quadratic monomials with a three generators $\varphi_{j}$ we have

$$
\psi_{1}=\varphi_{1}^{2}, \quad \psi_{2}=\varphi_{1} \varphi_{2}, \quad \psi_{3}=\varphi_{1} \varphi_{3}, \quad \psi_{4}=\varphi_{2}^{2}, \quad \psi_{5}=\varphi_{2} \varphi_{3}, \quad \psi_{6}=\varphi_{3}^{2} .
$$


Passing to homogeneous coordinates and their logarithmic derivatives in the formula

$$
\frac{\left\langle\psi_{1}, \ldots, \psi_{6}\right\rangle}{\left\langle\varphi_{1}, \varphi_{2}, \varphi_{3}\right\rangle^{4}}
$$

by Lemma 2.1 we arrive at different degree of homogeneity $\left.\right|^{1}$ of the polynomials in differential variables $g_{j}$ in the numerator and denominator of the considered quotient. We introduce the following notations:

$$
\begin{gathered}
g_{j} \stackrel{\text { def }}{=}\left(\log \frac{\psi_{j}}{\psi_{6}}\right)_{x} \Rightarrow g_{1}=2 g, \quad g_{2}=g+h, \quad g_{3}=g, \quad g_{4}=2 h, \quad g_{5}=h \\
g=f_{1}-f_{3}, \quad h=f_{2}-f_{3} ; q \quad f_{j} \stackrel{\text { def }}{=}\left(\log \varphi_{j}\right)_{x}, \quad j \in[3] .
\end{gathered}
$$

In terms of these notations, we employ Lemma 2.1 to obtain:

$$
\frac{\left\langle\psi_{1}, \psi_{2}, \ldots, \psi_{6}\right\rangle}{\left\langle\varphi_{1}, \varphi_{2}, \varphi_{3}\right\rangle^{4}}=-\left(\begin{array}{ccc}
g_{1} & \cdots & g_{5} \\
g_{1}^{\prime}+g_{1}^{2} & \cdots & g_{5}^{\prime}+g_{5}^{2} \\
g_{1}^{\prime \prime}+3 g_{1} g_{1}^{\prime}+g_{1}^{3}, & \cdots & g_{5}^{\prime \prime}+3 g_{1} g_{5}^{\prime}+g_{5}^{3}
\end{array}\right)\left(\begin{array}{cc}
g & h \\
g^{\prime}+g^{2} & h^{\prime}+h^{2}
\end{array}\right)^{-4} .
$$

Under an exponential approximation, the logarithmic derivatives are constant:

$$
\frac{\left\langle\psi_{1}, \psi_{2}, \ldots, \psi_{6}\right\rangle}{\psi_{1} \dot{\psi}_{2} \cdots \psi_{6}}=8(h-g)^{4}(h g)^{4}(2 h-g)(h-2 g)(g+h) ; \quad\left(\frac{\left(\left\langle\varphi_{1}, \varphi_{2}, \varphi_{3}\right\rangle\right)}{\varphi_{1} \varphi_{2} \varphi_{3}}\right)^{4}=(h-g)^{4}(g h)^{4},
$$

and hence

$$
\frac{\left\langle\psi_{1}, \psi_{2}, \ldots, \psi_{6}\right\rangle}{\left\langle\varphi_{1}, \varphi_{2}, \varphi_{3}\right\rangle^{4}} \approx 8(2 h-g)(h-2 g)(h+g) .
$$

In view of this we can make a conjecture on the multiplicity of the zeroes in the equation

$$
\left\langle\varphi_{1}, \varphi_{2}, \varphi_{3}\right\rangle=0 \Rightarrow\left\langle\psi_{1}, \psi_{2}, \ldots, \psi_{6}\right\rangle=0
$$

and on a polynomial in the sense of Lemma 1.1 nature of the answer for general functions $\varphi_{j}$, not necessarily exponential.

Thus, the Wronskians are replaced by the Vandermonde determinant for the exponential functions and the problem is reduced to an algebraic one. In particular, quotient (2.12) of the Wronskians is reduced in this case to the quotient of the Vandermonde determinants and this quotient is independent of the exponents in the functions $\varphi_{1}$ and $\varphi_{2}$. It is clear that the obtained invariance condition with respect to the exponents is only necessary for ensuring identities similar to 2.12 .

\section{DARBOUX TRANSFORMS AND FACTORIZATION}

In the case of monomials with three generators $\varphi_{1}^{i} \varphi_{2}^{j} \varphi_{3}^{k}, i+j+k=m \geqslant 2$, we fail to find an appropriate normalizing factor for Wronskian identities like 2.15). An exponential version of this problem was briefly considered in the end of the previous section. Basing on an arbitrary choice of the functions in identities similar to (2.12), as a first step, to the Darboux transforms of zero order, we add transformations described by the changes $\varphi \rightarrow \varphi^{\prime}-f \varphi$, that is, the Darboux transforms of the first order. The following version of a lemma known in the theory of integrable system hints which transformations can be employed instead of 1.2 in the considered problem:

Lemma 3.1. For the Wronskians $\left\langle\psi_{1}, \ldots, \psi_{m}\right\rangle \stackrel{\operatorname{def}}{=} \operatorname{det}\left(\partial_{x}^{k-1}\left(\psi_{j}\right)\right), j, k=1, \ldots, m$ on arbitrary $m \geqslant 2$ smooth functions $\psi_{j}(x)$ the following formula holds:

$$
\left\langle\psi_{1}, \ldots, \psi_{m}\right\rangle=\psi_{1}\left\langle\hat{\psi}_{2}, \ldots, \hat{\psi}_{m}\right\rangle, \quad \hat{\psi}_{j}=(D-f) \psi_{j}, \quad f=D \log \psi_{1} .
$$

\footnotetext{
${ }^{1}$ in 2.15 they coincide
} 
Proof. Expanding the determinants $\left\langle\psi_{1}, \ldots, \psi_{m}\right\rangle$ and $\left\langle\hat{\psi}_{2}, \ldots, \hat{\psi}_{m}\right\rangle$ over the last column and taking into consideration that $\hat{\psi}_{m}=(D-f)\left(\psi_{m}\right)$, we obtain two expressions as a differential operator of order $m-1$ acting on the function $\psi_{m}$ :

$$
\begin{aligned}
& A\left(\psi_{m}\right)=\left(a_{0} D^{m-1}+a_{1} D^{m-2}+\cdots+a_{m-1}\right)\left(\psi_{m}\right) \\
& \hat{A}\left(\psi_{m}\right)=\left(\hat{a}_{0} D^{m-2}+\hat{a}_{1} D^{m-3}+\cdots+\hat{a}_{m-2}\right)(D-f)\left(\psi_{m}\right)
\end{aligned}
$$

It is easy to see that the zero-spaces $\operatorname{ker} A$ and $\operatorname{ker} \hat{A}$ contain the functions $\psi_{1}, \ldots, \psi_{m-1}$ and therefore coincide. It remains to observe that a needed identity $a_{0}=\psi_{1} \hat{a}_{0}$ is equivalent to formula (3.1):

$$
\left\langle\psi_{1}, \ldots, \psi_{m-1}\right\rangle=\psi_{1}\left\langle\hat{\psi}_{2}, \ldots, \hat{\psi}_{m-1}\right\rangle,
$$

but with replacing $m$ by $m-1$. To complete the proof, we can use the induction in $m$ since as $m=2$ we have:

$$
\left\langle\psi_{1}, \psi_{2}\right\rangle=\psi_{1} \psi_{2}^{\prime}-\psi_{2} \psi_{1}^{\prime}=\psi_{1}(D-f) \psi_{2}=\psi_{1} \hat{\psi}_{2}
$$

An immediate corollary of Lemma 3.1 is the following theorem on factorization of an arbitrary differential operator specifying initial formula (1.1).

Theorem 3.1. Assume that given functions $\varphi_{1}, \ldots, \varphi_{n}$ form a basis of $n$-dimensional linear space ker $A$. Then

$$
\begin{gathered}
A=a_{0}\left(D-z_{n}\right)\left(D-z_{n-1}\right) \cdots\left(D-z_{1}\right), \quad z_{j}=\left[\log \left(w_{j} / w_{j-1}\right)\right]_{x}, \\
w_{j} \stackrel{\text { def }}{=}\left\langle\varphi_{1}, \ldots, \varphi_{j}\right\rangle, \quad j \in[n], \quad w_{0} \stackrel{\text { def }}{=} 1 .
\end{gathered}
$$

Proof. Rewriting formula (1.1) as $A(\varphi)=a_{0}\left\langle\varphi, \varphi_{1}, \ldots, \varphi_{n}\right\rangle$ and applying Lemma 3.1, we obtain:

$$
A(\varphi)=a_{0,1} A_{1} \circ\left(D-z_{1}\right)(\varphi), \quad z_{1}=\left(\log \varphi_{1}\right)_{x} \equiv\left[\log \left(w_{1} / w_{0}\right)\right]_{x},
$$

where the operator $A_{1}(\hat{\varphi})=\left\langle\hat{\varphi}, \hat{\varphi}_{2}, \ldots, \hat{\varphi}_{n}\right\rangle$ has order $n-1$ and we can apply the induction.

Employing Lemma 3.1 and Theorem 3.1, as in case 1.2 , we can reduce the order of the considered Wronskians. For instance, letting $\hat{\varphi}=\varphi^{\prime}-z \varphi, z=\left(\log \varphi_{3}\right)_{x}$, we obtain:

$$
\left\langle\varphi_{1}, \varphi_{2}, \varphi_{3}\right\rangle=\varphi_{3}\left\langle\hat{\varphi}_{1}, \hat{\varphi}_{2}\right\rangle, \quad \hat{\varphi}_{j}=\varphi_{j}^{\prime}-z \varphi_{j}, \quad j \in[2] .
$$

However, in the case, when the initial functions $\varphi_{j}, j \in[3]$, see Section 2, form a basis in ker $A$ for a given differential operator $A$, the transform $\varphi \rightarrow \hat{\psi}$ allows us, see, for instance, [1, to replace $^{1}$ the initial operator by the operator $\hat{A}$ with another order of the factors in factorization formula $(3.2)$.

We note that the main applications of the Darboux transforms for third order operators $A$, see [4], [5], are related with an anti-symmetric case:

$$
A+A^{*}=0, \quad A=D^{3}+a D+b \quad \Leftrightarrow \quad 2 b=a^{\prime},
$$

coinciding with the operator $L$ in formula $(2.2)$ for the squares of the eigenfunctions for the Schrödinger operators. This coincidence is not accidental and a relation between the Darboux transforms of zero and first orders, via the Ricatti equation for the logarithmic derivatives of the eigenfunctions deserves an additional study.

Finally we note that the Laplace transforms are also invertible Darboux transforms and algebraic studies of two-dimensional analogues of Wronskians, see, for instance, [2, related with these transforms give a chance to have useful contacts in this field with our colleagues from Ufa.

\footnotetext{
${ }^{1}$ in the problem on the eigenfunctions
} 


\section{ACKNOWLEDGMENTS}

We express our sincere gratitude to V.E. Adler and the participants of the seminar "Integrable systems" in Maykop city for useful remarks and an interest to our work.

\section{BIBLIOGRAPHY}

1. A.A. Allahverdyan. On Darboux transfors of Bessel functions // Vladikavkaz Math. J. 21:3, 5-13, (2019). (in Russian).

2. D.Demskoi, D.Tran Darboux integrability of determinant and equations for principal minors // Nonlinearity. 29:7, 36 (2014).

3. A.B. Shabat, M.Kh. Efendiev. On applications of Faà-di-Bruno formula // Ufimskij Matem. Zhurn. 9:3, 132-137 (2017). [Ufa Math. J. 9:3, 131-136 (2017).]

4. C. Verhoeven, M. Musette. Extended soliton solutions for the Kaup-Kupershmidt equation // J. Phys. A: Math. Gen. 34:11, 2515-2523 (2001).

5. M.C. Nucci. Pseudopotentials, Lax equations and Backlund transformations for nonlinear evolution equations // J. Phys. A: Math. Gen. 21:1, 73-79 (1988).

6. B.A. Dubrovin, V.B. Matveev, S.P. Novikov. Non-linear equations of Korteweg-de Vries type, finite-zone linear operators, and Abelian varieties // Uspekhi Matem. Nauk. 31:1(187), 55-136 (1976). [Russ. Math. Surv. 31:1, 59-146 (1976).]

Alina Albertovna Allahverdyan,

Adyghe State University,

Pervomayskaya str. 208,

385000, Maykop, Russia

E-mail: alinaallakhverdyan@mail.ru

Alexey Borisovich Shabat,

Adyghe State University,

Pervomayskaya str. 208,

385000, Maykop, Russia

E-mail: shabatab@mail.ru 